\title{
Real-time multitasking using ARM7 for elevator and camera with inbuilt embedded shell
}

\author{
K. PRASAD BABU ${ }^{1}$, SHAIK JHANI BASHA ${ }^{2}$
}

\author{
Assistant Professor, Electronics and Communication Engineering, GITAM School of Technology, Hyderabad, India ${ }^{1,2}$
}

\begin{abstract}
In this paper we are coming up with an embedded system with ARM7 processor controlling the operation of the elevator and camera. The microprocessor is programmed to run three tasks namely an elevator, a camera, and an embedded shell. The elevator and camera work together with the camera taking a snapshot of the occupants in the elevator every time the door opens. The tasks need precise timings which require employment of a real time operating system which provides determistic task switching's and precise outputs. This paper employs RTX real-time operating system for the three tasks to run concurrently.

The elevator is programmed in a way to accept simultaneous pressing of the entire floor buttons and is based on a UART system which makes display to be connected to it. The camera takes a photo as soon as the door is opened as it is given as a separate task there is no way the camera will fail to take a photo before the door is closed.

This paper also includes an embedded shell which can monitor and manipulate any memory location on the target board making it a powerful tool for debugging errors and can read and write data on to various memory locations in the processor. It can be used on any embedded system using an ARM7 processor giving it a wide range of applications.
\end{abstract}

Keywords : Multitasking, Embedded Shell, ARM7 processor, RTX.

\section{INTRODUCTION}

Elevator for secured buildings has been designed for a five floored building with a cellar. It has an inbuilt camera functionality which takes the snapshot of the occupants of the elevator every time the door is opened. This helps the security personnel of the building to know who is entering the building and leaving giving complete control of the movement of people in the building. As it does not have to run continuously like a CCTV camera, it saves a lot of disk space of the monitoring computer. The operation of the elevator has been mimicked using a UART which takes inputs from a general computer keyboard and does operations according to the commands. It has been programmed to take multiple commands, store them and execute them one after the other. The outputs of the elevator are demonstrated using the LEDs of the general I/O ports, the corresponding LED of the floor glows indicating that the elevator is in that particular floor. The text "OPEN" and "CLOSE" indicate the opening and closing of the elevator doors and as soon as the door opens the text "CAMERA ON" and the corresponding led that has been assigned to the camera blinks indicating that the camera takes a photo.

The embedded shell is a program designed to do various operations based on a set of instructions that have been pre assigned by the designer. It's similar to a command line interface which takes commands and displays the outputs on the UART. It can both read and write data from and to any device with a UART interface. Various commands have been assigned for flexible reading and writing of data.

Multitasking is an important feature found in most of the modern electronics. It increase the efficiency of the system and makes use of the processor perfectly. This has been incorporated in the elevator for predictable performance of the camera making sure that the camera does it job at all times and the control goes to it as soon as the door is opened and the door can be closed only after the camera does its job. This has been made possible by using RTX, a real time operating system which can give deterministic responses of the multiple tasks the processor has to handle.

\section{COMPONENTS DESCRIPTION:}

\section{ARM LPC2148}

Introduces LPC2148 microcontroller board based on a 16bit/32-bit ARM7TDMI-S CPU with real-time emulation and embedded trace support, that combine microcontrollers with embedded high-speed flash memory ranging from $32 \mathrm{kB}$ to $512 \mathrm{kB}$. A 128-bit wide memory interface and unique accelerator architecture enable 32-bit code execution at the maximum clock rate. For critical code size applications, the alternative 16-bit Thumb mode reduces code by more than $30 \%$ with minimal performance penalty. The meaning of LPC is Low Power Low Cost microcontroller. This is 32 bit microcontroller manufactured by Philips semiconductors (NXP).

Due to their tiny size and low power consumption, LPC2148 is ideal for applications where miniaturization is a key requirement, such as access control and point-ofsale.

\section{RTX (Real-Time Kernel)}

The RTX Real-Time Kernel is a real-time kernel for ARM7, ARM9, and Cortex-M devices. It allows you to create programs that simultaneously perform multiple functions or tasks. This is often required in an embedded application.

\section{Features}

- Royalty-free, deterministic RTOS with source code

- Designed for Cortex-M0, Cortex-M1, Cortex-M3, and ARM7, ARM9 
- High-Speed real-time operation with low interrupt latency

- Small footprint for resource constrained systems

- Flexible Scheduling: round-robin, pre-emptive, and collaborative

- Unlimited number of tasks with 255 priority levels

- Unlimited number of mailboxes, semaphores, mutex, and timers

- Support for multithreading and thread-safe operation

- Kernel aware debug support in MDK-ARM

- Dialog-based setup using $\mu$ Vision Configuration Wizard

\section{Benefits}

While it is certainly possible to create real-time programs without an RTOS (by executing one or more functions or tasks in a loop), there are numerous scheduling, maintenance, and timing issues that an RTOS like the RTX Real-Time Kernel solves for you.

\section{PROPOSED SYSTEM}

In the proposed work tasks are set through the program for elevator, camera and embedded shell. The block diagram of the proposed system is shown in Fig. 1 below. The elevator and camera work together with the camera taking a snapshot of the occupants in the elevator every time the door opens which makes the tasks interdependent and time critical. The tasks need precise timings which requires employment of a real time operating system which provides determistic task switchings and precise outputs. This paper employs RTX real-time operating system for the three tasks to run in real time with precision.

The elevator is programmed in a way to accept simultaneous pressing of all the floor buttons and is based on a UART system to connect it to the display to be connected to it. The camera takes a photo as soon as the door is opened. As it is given as a separate and time critical task, there is no way the camera will fail to take a photo before the door is closed.

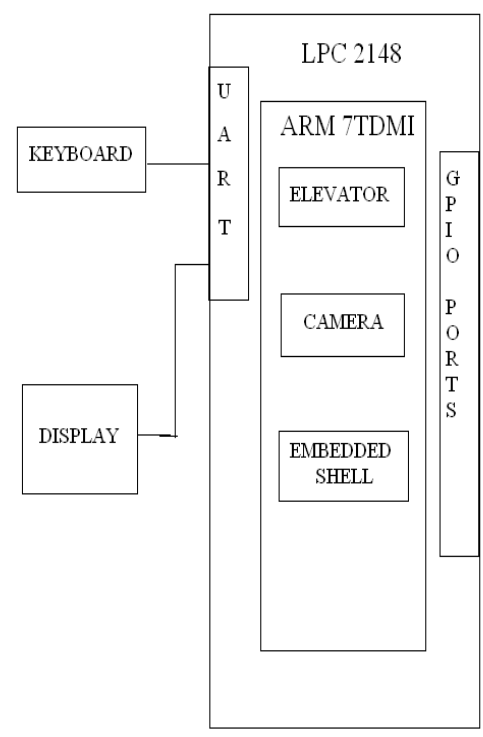

Fig.1 Block diagram of the system

\section{IMPLEMENTATION}

In the prototype of the system, elevator for secured buildings has been designed for a five floored building with a cellar. It has an inbuilt camera functionality which takes the snapshot of the occupants of the elevator every time the door is opened. This helps the security personnel of the building to know who is entering the building and leaving giving complete control of the movement of people in the building. As it does not have to run continuously like a CCTV camera it saves a lot of disk space of the monitoring computer. The operation of the elevator has been mimicked using a UART which takes inputs from a general computer keyboard and does operations according to the commands. It has been programmed to take multiple commands, store them and execute them one after the other. The outputs of the elevator are demonstrated using the leds of the general I/O ports, the corresponding LED of the floor glows indicating that the elevator is in that particular floor. The text "OPEN" and "CLOSE" indicate the opening and closing of the elevator doors and as soon as the door opens the text "CAMERA ON" and the corresponding led that has been assigned to the camera blinks indicating that the camera takes a photo.

The embedded shell is a program designed to do various operations based on a set of instructions that have been pre assigned by the designer. It's similar to a command line interface which takes commands and displays the outputs on the UART. It can both read and write data from and to any device with a UART interface. Various commands have been assigned for flexible reading and writing of data. Along with these there are other useful commands which include

1. Help -displays the available commands and usage of them.

2. R.b 0x8digit address - reads a byte of data from the given address.

3. R.w 0x8digit address - reads a word of data from the given address.

4. R.1 0x8digit address -reads a long (8 bytes) of data from the given address.

5. w.b 0x8digit address data -writes a byte of data from the given address.

6. w.w 0x8digit address data -writes a word of data from the given address.

7. w.1 0x8digit address data -writes a long ( 8 bytes) of data from the given address.

8. Ledn - switches on an LED.

9. Ledf - switches off an LED.

10. Ledb - blinks an LED.

11. Reset - resets the board.

12. Halt - hangs the board.

Multitasking is an important feature found in most of the modern electronics. It increase the efficiency of the system and makes use of the processor perfectly. This has been incorporated in the elevator for predictable performance of the camera making sure that the camera does it job at all times and the control goes to it as soon as the door is opened and the door can be closed only after the camera is done with its job. This has been made possible by using 
RTX, a real time operating system which can give having message alert system through a GSM module. Real deterministic responses of the multiple tasks the processor time display of the photo taken by the camera can be done has to handle.

\section{FLOWCHART OF THE PROCESS}

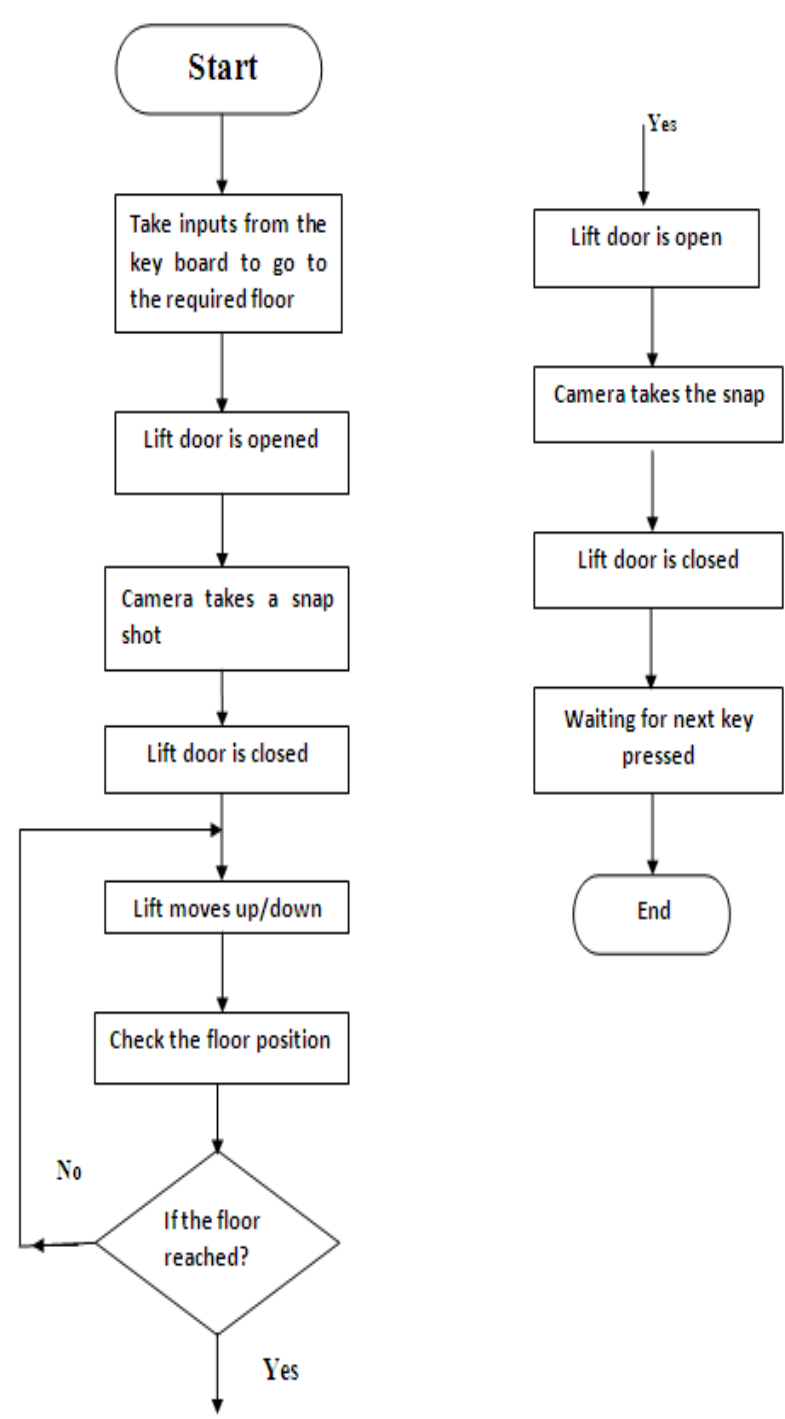

The programs written in the Assembly langrage and the corresponding hex codes are developed using the assembler. The hex code is finally dumped into the microcontroller using the Top win Universal Programmer. In order to give the commands using RTX and Embedded shell are used. Microcontroller controls the devices through relays depending on the code received by the RTX. The above figure shows the receiver side.

\section{CONCLUSION}

Multitasking on ARM7 is thus implemented successfully using an elevator and camera as tasks, the tasks run successfully with their assigned priorities which helps in obtaining a security enabled elevator for buildings. The elevator is capable of taking three inputs at a time and processes them in a first come first serve basis. The proposed system can be extended to include more tasks like image processor and alarm to make it more secured system. Alternately the security can be enhanced by by appropriately accessing the images and transmitting them to the display unit located in the control room.

\section{REFERENCES}

[1] J. Kumagai and S. Cherry, "Sensors and sensibili-ty," IEEE Spectr ., vol. 41, no. 7, pp. 22-26, July 2004.

[2] Grolle, S. CCTV to prevent crime? page(s): 3-12, August 2008. ISBN: 978-3-640-35488-7.

[3] Q.Zong,W.J.Liu,H.B.Xue: The application of GPRS to the remote elevator fault diagnosis system(Chinese)[J]. Elevator World Inc,2005,53(9),pp.138-147.

[4] Design of ARM-based Elevator Main Controller Zhang Rucheng1,Wang Guangsheng1,Zhang Lei2(1.College of Electronic Information \&amp; Control Engineering, Beijing University of Technology, Beijing 100124;2.Fengtai District Special Equipment Inspection \&amp; Testing Institute, Beijing 100073)

[5] Shouqian $\mathrm{Yu}$, Weihai Chen, Li Li ; Jianglei Qin "Development of ARM-based Embedded System for Robot Applications" IEEE XPLORE, December, 2006.

[6] Michael Barr, "Programming Embedded Systems in C and C++", America: O'Reilly, 2001

[7] L. G. Zhou, . Foundation and Practice for ARM Microprocessors, Beijing: Beijing University of Aeronautics and Astronautics Press, 2003

[8] Gunar Schirner, Gautam Sachdeva, Andreas Gerstlauer, Rainer Domer "Modeling, Simulation and Synthesis in an Embedded Software Design Flow for an ARM Processor" Center for Embedded Computer Systems University of California, Irvine, May 2006.

\section{BIOGRAPHIES}

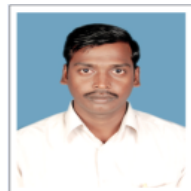

K. Prasad Babu, presently working in the department of Electronics and Communication Engineering in GITAM University. His areas of interest are Embedded Systems, Digital Systems and VLSI. He has 5.6 years of teaching experience.

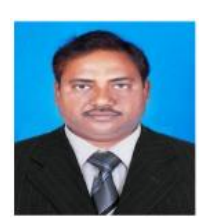

S Haik Jhani Bhasha, presently working in the department of Electronics and Communication Engineering in GITAM University. His areas of interests are Embedded System, Micro Electronics and Renewable energy Sources. He has 14 years of teaching experience. He is a life member of ISTE. 